SPICA Workshop, $04011(2009)$

DOI:10.1051/spica/200904011

(C) Owned by the authors, published by EDP Sciences, 2009

This is an Open Access article distributed under the terms of the Creative Commons Attribution-Noncommercial License, which permits unrestricted use, distribution, and reproduction in any noncommercial medium, provided the original work is properly cited.

\title{
LUMINOUS BURIED AGNS IN ULTRALUMINOUS INFRARED GALAXIES AND THE ORIGIN OF GALAXY DOWNSIZING
}

\section{Imanishi}

Division of Optical and Infrared Astronomy, National Astronomical Observatory of Japan, 2-21-1, Osawa, Mitaka 181-8588, Japan

\section{ABSTRACT}

We present the results of our systematic infrared 2.5$35 \mu \mathrm{m}$ low-resolution spectroscopy of nearby ultraluminous infrared galaxies (ULIRGs) at $z<0.3$, using Subaru, AKARI, and Spitzer. We detected the signatures of optically-elusive, but intrinsically luminous, buried AGNs in a significant fraction of ULIRGs classified optically as non-Seyferts. We found that the energetic importance of AGNs increases with increasing galaxy infrared luminosity, suggesting that the AGN-starburst connections are luminosity dependent. This result may be related to the stronger AGN feedback scenario in currently more massive galaxy systems, as the origin of the galaxy downsizing phenomenon. It is crucially important for SPICA to investigate the AGN-starburst connections in the early universe at $z>1$, when both star-formation and AGN activity show peaks.

Key words: Galaxies: formation - Galaxies: active - galaxies: ISM - Galaxies: nuclei - Galaxies: Seyfert - Galaxies: starburst - Infrared: galaxies - Missions: SPICA

\section{INTRODUCTION}

Ultraluminous infrared galaxies (ULIRGs) are the population which displays a spectral energy distribution dominated by infrared emission and has absolute infrared luminosity with $\mathrm{L}_{\mathrm{IR}}>10^{12} \mathrm{~L}_{\odot}$ (Sanders \& Mirabel, 1996). This indicates that in ULIRGs, (1) very luminous energy sources are present, but hidden behind dust, (2) the bulk of the energetic radiation from the hidden energy sources is absorbed by the surrounding dust, and (3) the heated dust re-emits this energy as infrared dust radiation. The dust-obscured hidden energy sources of ULIRGs can be nuclear fusion inside rapidly formed stars (i.e., starburst activity) and/or active mass accretion onto a central supermassive black hole $(\mathrm{SMBH})$ with a mass $>10^{6} \mathrm{M}_{\odot}$ (i.e., AGN activity). Since the ULIRG population contributes very importantly to the comic infrared radiation density at $z>1$, the crucial epoch of galaxy formation and SMBH mass growth (Caputi et al., 2007), understanding the hidden energy sources of ULIRGs is essential to clarify the AGN-starburst connections in the dust-obscured portion of the universe. Since distant ULIRGs are generally faint, detailed studies of nearby ULIRGs at $z<0.3$ have played an important role in understanding the nature of the ULIRG population.

If a luminous AGN is present hidden behind torusshaped dusty medium, the so-called narrow line regions (NLRs), photo-ionized by AGN's radiation, should develop along the torus axis, above a torus scale height, and NLRs will be visible from all directions. Since an ionizing spectrum is different between an AGN and stars, optical emission line flux ratios are also different between NLRs in an AGN and clouds in a starburst galaxy. Such an AGN as obscured by torus-shaped dusty medium is classified optically as a Seyfert 2 and so is easily distinguishable from a normal starburst galaxy through optical spectroscopy. However, since the nuclear regions of ULIRGs are very dusty (Sanders \& Mirabel, 1996), AGNs resident in the majority of ULIRGs may be buried (i.e. obscured by dust along virtually all sightlines). It is fundamental to understand such optically elusive buried AGNs in the nuclei of ULIRGs.

Low-resolution $(\mathrm{R} \sim 100)$ infrared $2.5-35 \mu \mathrm{m}$ spectroscopy is an effective means to study optically elusive buried AGNs, because dust extinction is small $\left(<0.06 \mathrm{~A}_{\mathrm{V}}\right)$. More importantly, normal starburst and AGN emission are clearly distinguishable using infrared $2.5-35 \mu \mathrm{m}$ spectra. First, in a normal starburst galaxy, large equivalent width polycyclic aromatic hydrocarbon $(\mathrm{PAH})$ emission features should always be seen, regardless of dust extinction of the starburst, while a pure AGN emits a PAH-free continuum (Imanishi \& Dudley, 2000). Next, in a normal starburst, where the stellar energy sources and dust are spatially well mixed (Figure 1, left), there is an upper limit for the optical depths of dust absorption features in infrared spectra. Optical depths can be arbitrarily large in a buried AGN, where the energy source is more centrally concentrated than the surrounding dust (Figure 1, right) (Imanishi \& Maloney, 2003; Imanishi et al., 2006; 2007).

In this manuscript, we present the results of groundbased Subaru IRCS infrared 2.8-4.1 $\mu \mathrm{m}$ spectroscopy, spacebased AKARI IRC 2.5-5 $\mu \mathrm{m}$ spectroscopy, and spacebased Spitzer IRS 5-35 $\mu \mathrm{m}$ spectroscopy of a sample of nearby ULIRGs with $z<0.3$ classified optically as nonSeyferts (i.e., LINERs, HII-regions, and unclassified), selected from the IRAS 1 Jy sample (Kim \& Sanders, 1998). 


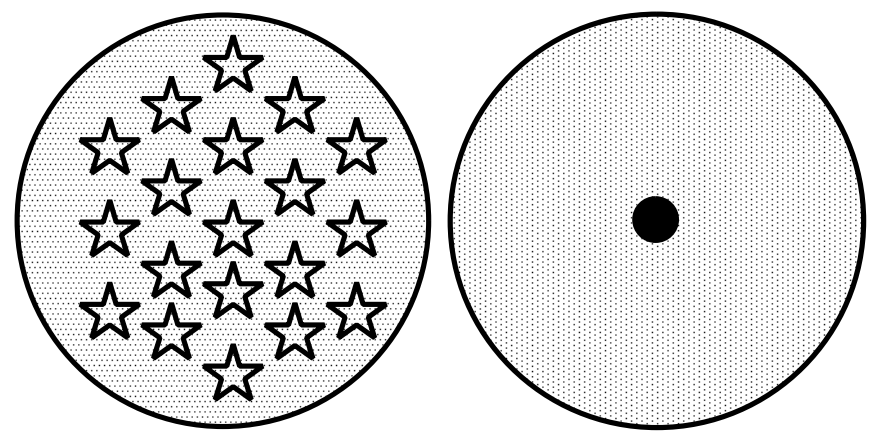

Figure 1. Geometry of energy sources and dust. (Left): Normal starburst. Stellar energy sources (open stars) and dust are spatially well mixed. (Right): Buried AGN. The energy source (= a mass accreting supermassive black hole; filled circle) is spatially very compact and is more centrally concentrated than the surrounding dust.

\section{Results And Discussion}

Figures 2, 3, and 4 present Subaru, AKARI, and Spitzer infrared spectra of selected ULIRGs at $z<0.3$, respectively (Imanishi et al., 2006; 2007; 2008).

\subsection{Weakly-OBSCUREd Starbursts AND BURIEd AGNs}

PAH emission, a probe of starbursts, is detected in almost all of the observed ULIRGs. It is likely that modestlyobscured $\left(\mathrm{A}_{\mathrm{V}}<15 \mathrm{mag}\right)$ starbursts are responsible for the detected PAH emission, because the effects of dust extinction for the PAH emission are small if $\mathrm{A}_{\mathrm{V}}<15 \mathrm{mag}$. The observed PAH to infrared luminosity ratios are substantially smaller than those found in known starburst galaxies with modest dust obscuration, suggesting that the detected modestly-obscured starbursts alone cannot account for the bulk of the large ULIRG's luminosities. The luminosity should come from non-PAH emitting AGNs and/or highly obscured $\left(\mathrm{A}_{\mathrm{V}}>>15 \mathrm{mag}\right)$ starbursts, where PAH emission is substantially flux-attenuated. These two scenarios could be distinguished by comparing the equivalent widths of emission and absorption features in infrared spectra (Imanishi et al., 2006; 2007; 2008).

Based on low PAH equivalent widths and strong dust absorption features (see $\S 1$ ), luminous buried AGN signatures were found in roughly half of the observed ULIRGs classified optically as non-Seyferts (Imanishi et al., 2006; $2007 ; 2008)$. We also found that (1) the total amount of nuclear dust is substantially larger in buried AGNs in optically non-Seyfert ULIRGs than Seyfert AGNs surrounded by torus-shaped dusty medium, and (2) ULIRGs with both warm (IRAS $25 \mu \mathrm{m}$ to $60 \mu \mathrm{m}$ flux ratio $>0.2$ ) and cool $(<0.2)$ far-infrared colors display luminous buried AGN signatures. Although Seyfert AGNs usually display warm far-infrared colors, the cooler far-infrared colors of buried AGNs can naturally be explained by a larger amount of nuclear dust, where contribution from outer, cooler dust
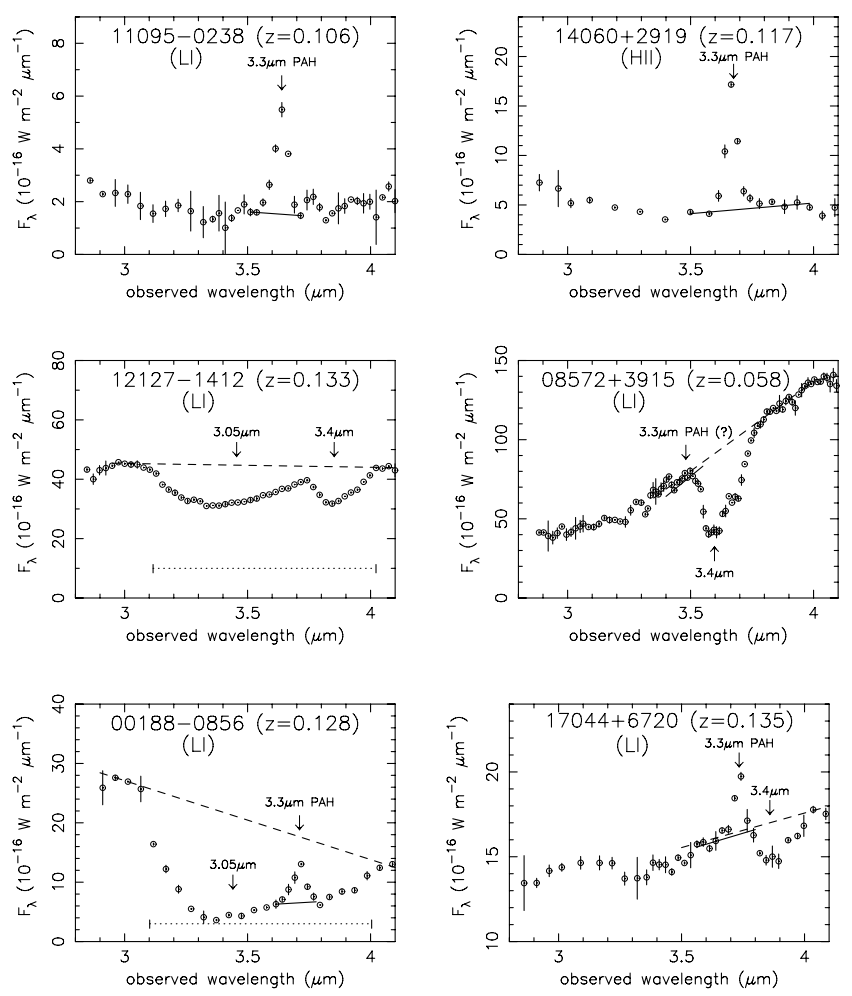

Figure 2. Examples of ground-based infrared 2.8-4.1 $\mu \mathrm{m}$ lowresolution $(R \sim 100)$ spectra of nearby ULIRGs, obtained with Subaru IRCS (Imanishi et al., 2006). The observed ULIRGs are limited to $z<0.15$, because above this redshift, the energy diagnostic based on rest-frame 3-4 $\mu \mathrm{m}$ spectra (Imanishi \& Dudley, 2000) is hampered, due to Earth's atmospheric absorption. Top : Starburst-dominated ULIRGs. Strong, large equivalent width PAH emission at rest-frame $3.3 \mu \mathrm{m}$ and nondetectable dust absorption features are seen. Middle : ULIRGs dominated by buried AGNs, with no detectable starbursts. No $3.3 \mu \mathrm{m}$ PAH emission and strong absorption features at restframe $3.4 \mu m$ by bare carbonaceous dust and/or at $3.1 \mu m$ (3.05 $\mu \mathrm{m})$ by ice-covered dust are detected. Bottom : ULIRGs with detectable starbursts and buried AGNs. PAH emission is seen, but its equivalent width is low. "LI" and "HII" mean ULIRGs classified optically as LINERs and HII-regions, respectively. The dotted line indicates the wavelength range where the broad $3.1 \mu \mathrm{m}$ ice absorption feature is strong. The solid and dashed lines are the continuum levels adopted to measure the strengths of $3.3 \mu \mathrm{m}$ PAH emission and dust absorption features, respectively.

component to the infrared radiation becomes more important.

\subsection{Dependence on GalaXy infRARED Luminosity}

By separating galaxies into those with $\mathrm{L}_{\mathrm{IR}}<10^{12} \mathrm{~L}_{\odot}$, $10^{12} \mathrm{~L}_{\odot} \leq \mathrm{L}_{\mathrm{IR}}<10^{12.3} \mathrm{~L}_{\odot}$, and $\mathrm{L}_{\mathrm{IR}} \geq 10^{12.3} \mathrm{~L}_{\odot}$, we found in Figure 5 that the buried AGN fraction clearly increases with increasing galaxy infrared luminosity, suggesting that the AGN-starburst connections are luminosity dependent. 

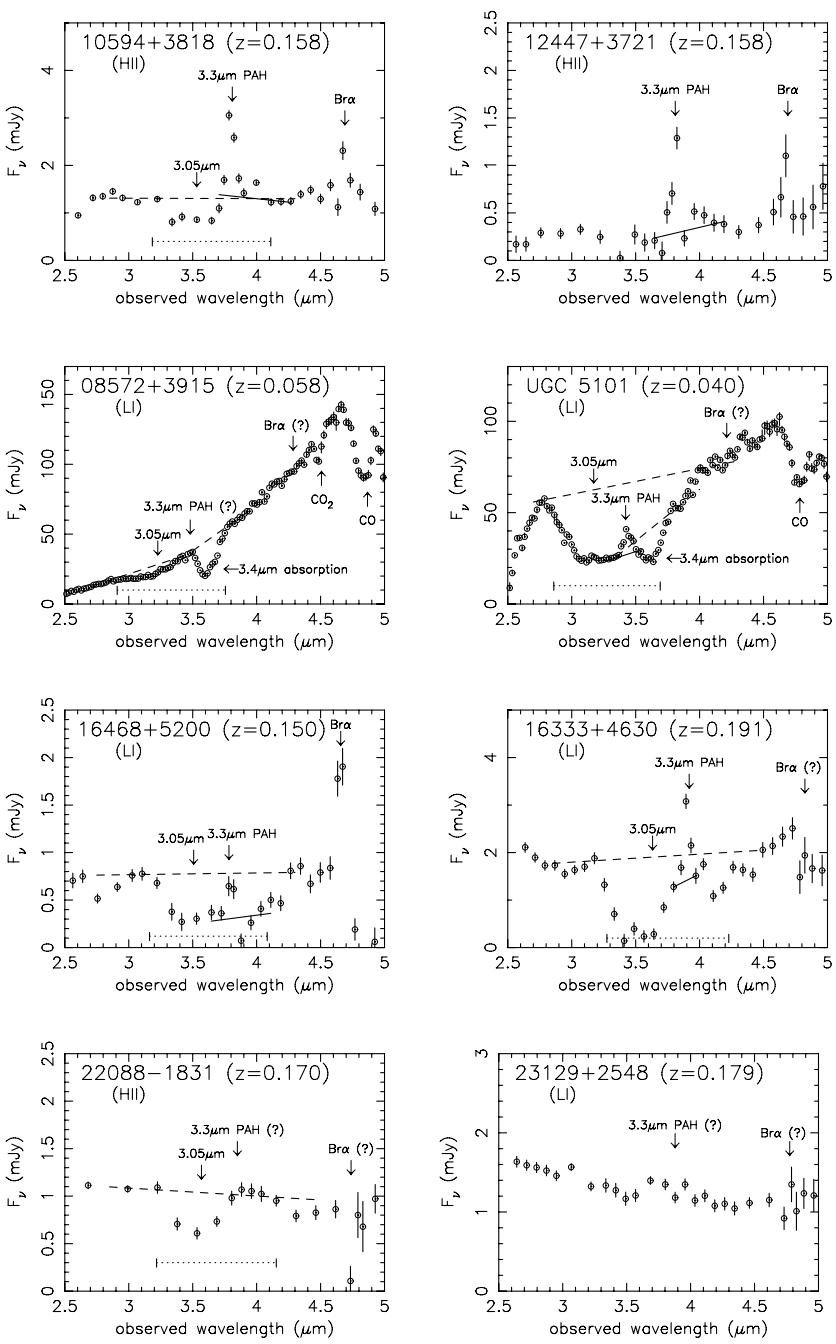

Figure 3. Examples of AKARI IRC 2.5-5 $\mu \mathrm{m}$ low-resolution $(R \sim 100)$ spectra of ULIRGs (Imanishi et al., 2008). With no atmospheric absorption effects for AKARI IRC spectroscopy, energy sources of ULIRGs at $z>0.15$ can be diagnosed. Top : ULIRG whose emission is explained only with starburst activity. Lower: ULIRGs displaying signatures of luminous buried AGNs. Symbols are the same as in Figure 2. $\mathrm{CO}$ and $\mathrm{CO}_{2}$ absorption features are indicated for detected sources. The expected wavelength of the Bra emission (rest-frame $4.05 \mu \mathrm{m}$ ) is also shown.

\subsection{BURIED AGNs AND GALAXY DOWNSIZING}

For buried-AGN-type ULIRGs with low PAH equivalent widths, the observed infrared 2.5-35 $\mu \mathrm{m}$ emission should be dominated by PAH-free, hot dust emission that is heated by AGNs. We quantitatively found that the intrinsic buried AGN luminosities can be up to $\sim 10^{12} \mathrm{~L}_{\odot}$, accounting for a significant (if not dominant) fraction $(<50 \%)$ of the observed large infrared luminosities of ULIRGs (Imanishi et al., 2008; Imanishi, 2009). If the remaining infrared luminosities are due to star formation (including highlyobscured $A_{V}>15$ mag one), then the star formation
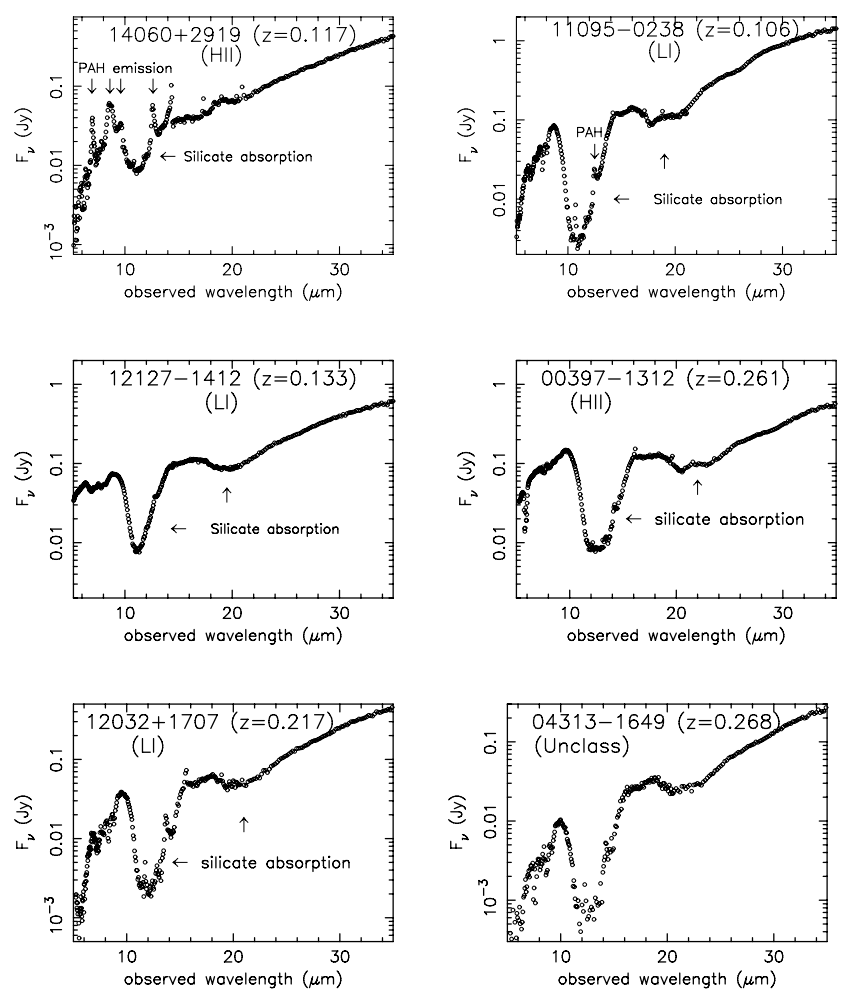

Figure 4. Infrared 5-35 $\mu \mathrm{m}$ low-resolution $(R \sim 100)$ spectra of ULIRGs obtained with Spitzer IRS (Imanishi et al., 2007). Top Left : Starburst-dominated ULIRG. The equivalent width of the PAH emission is large, and the optical depth of the silicate dust absorption feature at rest-frame $9.7 \mu \mathrm{m}$ is modest, within a range explained by the mixed dust/source geometry (Figure 1, left). Top Right and Lower: ULIRGs with luminous buried AGN signatures. No detectable or very weak PAH emission and strong silicate dust absorption features are detected. The optical depth of the $9.7 \mu \mathrm{m}$ silicate dust absorption feature exceeds the maximum value achieved by the mixed dust/source geometry (Figure 1, left). Symbols are the same as in Figure 2. "Unclass" refers to an optically-unclassified ULIRG.

rates must be high and many stars will be formed in the future. Summarizing, buried AGNs are relatively more important energetically in currently more infrared luminous galaxies, and these galaxies will evolve into more massive galaxies with larger stellar masses in the future.

Recently, the so-called galaxy downsizing phenomenon has been widely discussed, where more massive galaxies with currently larger stellar masses have finished their major star formation at an earlier cosmic age (e.g., Bundy et al., 2005). It is proposed that in currently more massive galaxies, AGN feedback has been stronger in the past, heating and/or expelling gas in host galaxies and suppressing further star formation on a shorter time scale (e.g., Bower et al., 2006). Buried AGNs surrounded by a large amount of gas and dust, rather than already visible AGNs, can have particularly strong feedback. The galaxy stellar mass above which buried AGNs become en- 


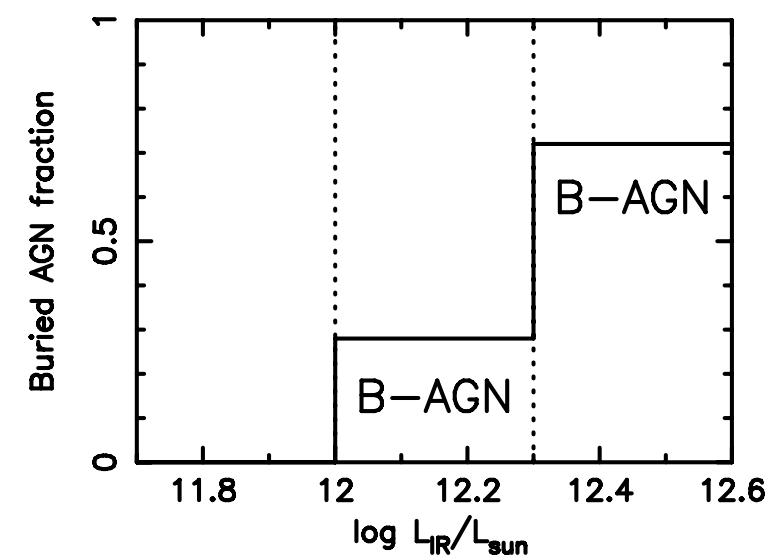

Figure 5. Fraction of sources with clearly detectable buried AGN signatures as a function of galaxy infrared luminosity (Imanishi, 2009).

ergetically important (i.e., ULIRGs) is several $\times 10^{10} \mathrm{~L}_{\odot}$. This roughly corresponds to the mass which separates red, massive galaxies and blue, less massive galaxies in the local universe (Kauffmann et al., 2003). Figure 6 illustrates this picture. Our results may be related to the AGN feedback scenario as the origin of the galaxy downsizing phenomenon.

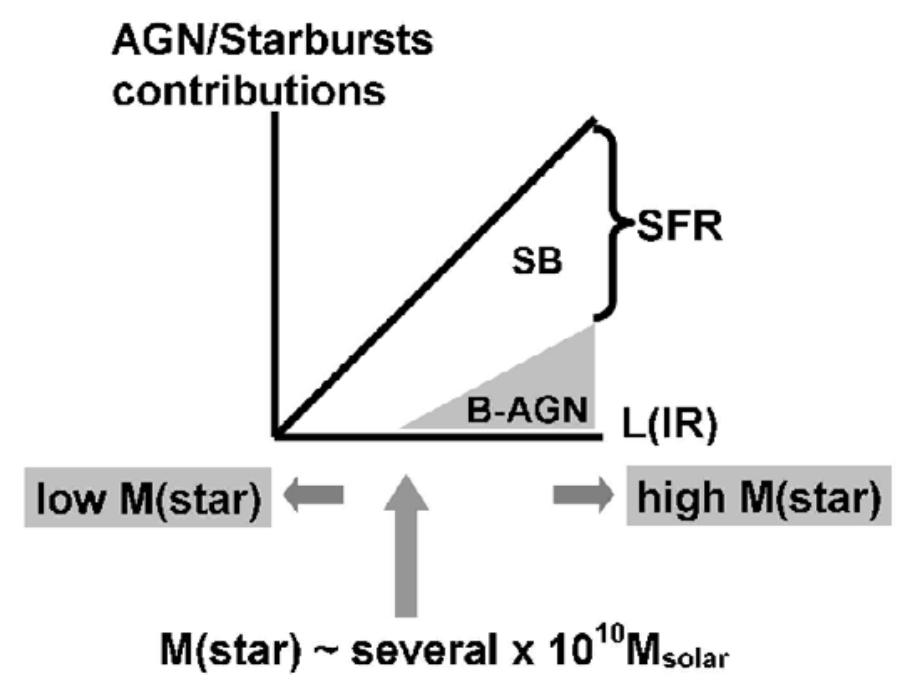

Figure 6. Schematic diagram of the energetic importance of buried $A G N s$ (B-AGN) and starbursts (SB) as a function of galaxy infrared luminosity. SFR means star formation rate. In galaxies with higher infrared luminosities, the energetic importance of buried AGNs is relatively higher, and higher SFRs suggest that these galaxies will evolve into more massive galaxies with larger stellar masses. The eventual galaxy stellar mass above which buried AGNs become important is about several $\times$ $10^{10} M_{\odot}$.

\subsection{Extension to the EARLy Universe USING SPICA}

With SPICA, we can apply this study to distant ULIRGs at $z>1$, when the star formation history and AGN space density show peaks. This is the crucial epoch to understand galaxy formation, SMBH mass growth, the interplay between AGN and starburst, and buried AGN feedbacks to host galaxies. For this purpose, low-resolution spectroscopy at $>6-50 \mu \mathrm{m}$ in the observed frame is needed. SAFARI and MIRACLE are clearly the best choice.

Although Spitzer IRS spectroscopy has been made for ULIRGs at $z>1$, the targets have been limited only to very bright sources with $>900 \mu \mathrm{Jy}$ at $\lambda=24 \mu \mathrm{m}$. A strong bias is unavoidable. Hence, the main targets for SPICA will be fainter, general ULIRGs. Galaxies brighter than $60 \mu \mathrm{Jy}$ at $\lambda=24 \mu \mathrm{m}$ could account for the bulk of the cosmic infrared background emission (Dole et al., 2006), and so can be targets. However, because of the $(1+z)$ dimming effect, these sources will be mostly at $z \sim 1$. Unbiased ULIRGs at $z=2-4$ must be selected based on infrared luminosity.

By applying our successful energy diagnostic methods to distant ULIRGs at $z>1$, we can comprehensively understand the AGN-starburst connections in the dust obscured portion of the universe.

\section{Summary}

Our infrared 2.5-35 $\mu \mathrm{m}$ low-resolution spectroscopy has shown that optically elusive, but intrinsically luminous buried AGNs are common in optically non-Seyfert ULIRGs in the local universe, and that the importance of buried AGNs increases with increasing galaxy infrared luminosity. Using SPICA, we can extend this study to $z>1$, the major epoch of galaxy formation and SMBH mass growth, in order to understand the AGN-starburst connections in the dust-obscured portion of the universe.

\section{REFERENCES}

Bower, R. G., et al. 2006, MNRAS, 370, 645

Bundy, K., Ellis, R. S., \& Conselice, C. J. 2005, ApJ, 625, 621

Caputi, K. I., et al. 2007, ApJ, 660, 97

Dole, H., et al. 2006, A\&A, 451, 417

Imanishi, M. 2009, ApJ, 694, 751

Imanishi, M., \& Dudley, C. C. 2000, ApJ, 545, 701

Imanishi, M., Dudley, C. C., \& Maloney, P. R. 2006, ApJ, 637,114

Imanishi, M., Dudley, C. C., Maiolino, R., Maloney, P. R.,

Nakagawa, T., \& Risaliti, G. 2007, ApJS, 171, 72

Imanishi, M., \& Maloney, P. R. 2003, ApJ, 588, 165

Imanishi, M., Nakagawa, T., Ohyama, Y., Shirahata, M.,

Wada, T., \& Onaka, T. 2008, PASJ, 60, S489

Kauffmann, G., et al. 2003, MNRAS, 341, 54

Kim, D. -C., \& Sanders, D. B., 1998, ApJS, 119, 41

Sanders, D. B., \& Mirabel, I. F. 1996, ARA\&A, 34, 749 